records were reviewed. Quality of care parameters; HCC screening and management of cirrhotic complications (secondary prophylaxis for variceal bleeding with Beta-blocker, abdominal paracentesis in the patient with ascites, antibiotic for secondary prevention of SBP, prevention of hepatic encephalopathy) were measured for the proportion of cases where the patient received recommended care according to AASLD practice guidelines. Costs and outcome were analyzed. Results were reported as total outcome and subgroup outcomes.

Results For the 1,180 cirrhotic patients, 30\% were followed up at gastroenterology out-patient clinic (GI), 33.4\% at general internal medicine outpatient clinic (MED), and 36.6\% at both clinics. Quality of care parameters were poorly met the standard recommendations especially in MED group (figure 1). There were 223 deaths occurred $(18.8 \%$ all-cause mortality). The average direct cost was $\$ 1,766$ per patient-year. The incremental cost-effectiveness ratio (ICER), demonstrated the superiority of GI group over MED group, was $\$ 16.7$ per one lifesaving per year (figure 1).

Conclusions Despite a high economic burden and mortality rate, current practice in cirrhotic patients in this study were poorly met the standard recommendations according to AASLD guidelines. The results founded in this study were the first data established and were relatively similar to those previous data from the US. The outcomes from this study led to

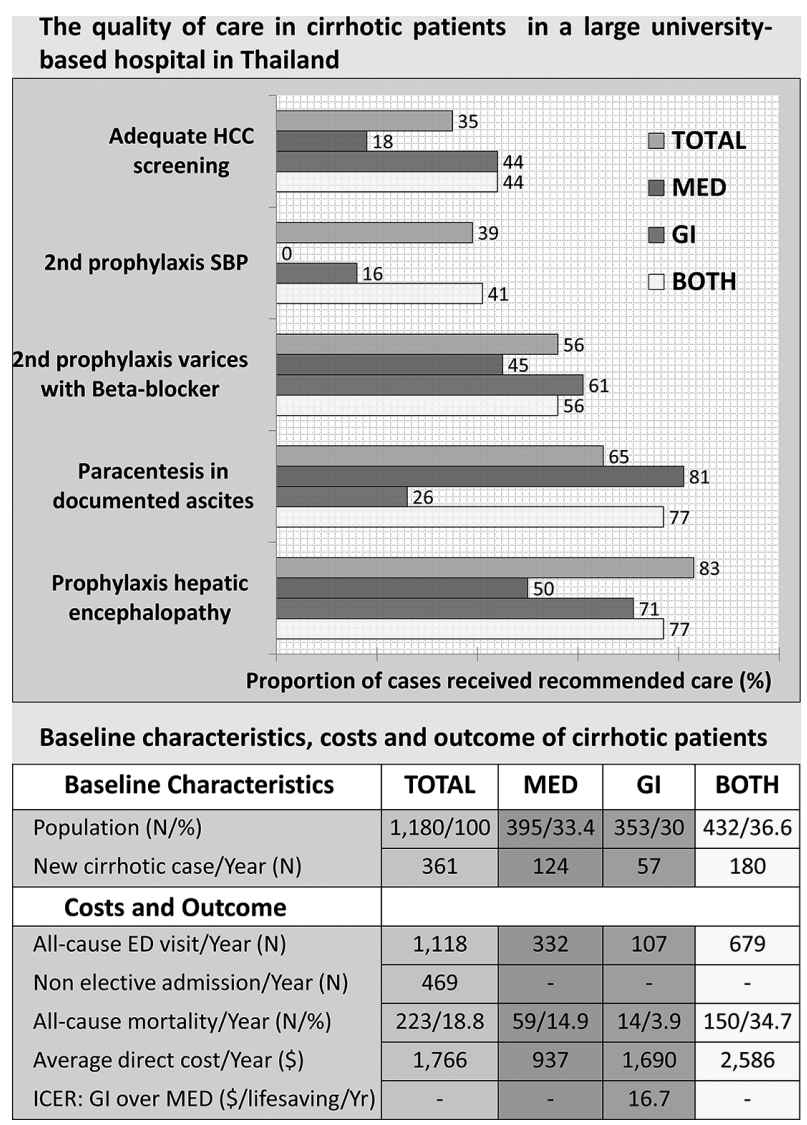

Abstract IDDF2019-ABS-0076 Figure 1 the next study 'A quality improvement initiative for achieving standard of care in cirrhosis' which is being conducted in Phramongkutklao hospital, Thailand.

\section{IDDF2019-ABS-0077 PERCUTANEOUS ENDOBILIARY RADIOFREQUENCY ABLATION AND SELF- EXPANDABLE METAL STENT PLACEMENT VERSUS STENT PLACEMENT ALONE FOR MALIGNANT BILIARY OBSTRUCTION}

Wei Cui ${ }^{*}$, Jiaping Li. The First Affiliated Hospital of SYSU, China

\subsection{6/gutjnl-2019-IDDFabstracts. 255}

Background To retrospectively evaluate the added benefit of adding endobiliary radiofrequency ablation (EB-RFA) to biliary metal stent placement for patients with malignant biliary obstruction (MBO).

Methods From November 2013 to June 2018, 163 patients with MBO who had undergone percutaneous EB-RFA and stent placement (RFA-stent group, $\mathrm{n}=90$ ) or stent placement only (Stent group, $\mathrm{n}=73$ ) were included. According to the causes of biliary obstruction, the two treatment groups were divided into Cholangiocarcinoma Subgroup, Pancreatic carcinoma Subgroup and Remaining disease Subgroup. Overall survival(OS), primary and secondary stent patency (PSP, SSP), complications between the two groups were compared, and the prognostic factors of OS, PSP and SSP were analyzed.

Results RFA-Stent group resulted in better median OS (7.0 vs 4.5 months, $\mathrm{P}=0.034)$, PSP $(8.0$ vs 5.1 months, $\mathrm{P}=0.003)$ and SSP $(9.8$ vs 5.1 months, $\mathrm{P}<0.001)$ than Stent group (figure 1). In Cholangiocarcinoma subgroup, the median OS in two groups was 8.0 months and 4.7 months, respectively, $\mathrm{P}=0.791$; The median PSP and SSP in RFA-Stent group were longer than that Stent group( PSP: 8.0 months vs 6.0 months, $\mathrm{P}=0.024$, respectively; SSP: 9.0 months vs 6.0 months, respectively, $\mathrm{P}<0.001$ ) (figure 2). In Pancreatic carcinoma subgroup, RFA-Stent group resulted in better median $\mathrm{OS}(8.5$ vs 4.0 months, $\mathrm{P}=$ $0.006)$, PSP (8.5 vs 4.5 months, $\mathrm{P}=0.021)$ and SSP (9.8 vs 4.5 months, $\mathrm{P}=0.004$ ) than Stent group(figure 3 ). There was no statistical difference in OS, PSP and SSP between the two groups in Remaining subgroup(figure 4). The complications were comparable between the two groups. Multivariate analysis showed that treatment methods, performance status score, postoperative combination therapy, obstruction etiology, obstruction location type, preoperative bilirubin level and postoperative bilirubin decreased value were independent prognostic factors for survival. Treatment methods, previous treatment for primary disease and preoperative bilirubin level were independent prognostic factors for PSP and SSP.

Conclusions RFA-Stent confers therapeutic benefits in patients with unresectable $\mathrm{MBO}$, especially in those patients with pancreatic carcinoma. 